\title{
Modeling of Functional Properties of Porous Shape Memory Alloy
}

\author{
Aleksandr E. Volkov ${ }^{1}$, Margarita E. Evard ${ }^{1, a}$ and Elizaveta N. Iaparova ${ }^{1}$ \\ ${ }^{1}$ Saint Petersburg State University, 198504 Saint Petersburg, Russia
}

\begin{abstract}
A model accounting for the microstructure of porous TiNi shape memory alloy samples fabricated by selfpropagating high temperature synthesis has been proposed for simulation of their functional-mechanical properties. Structural elements of a porous sample have been approximated by curved beams. An analysis of shapes and sizes of pores and ligaments permitted to identify characteristic sizes of the beams. A mathematical object consisting of rigidly connected small curve beams has been considered. The stress-strain state of a beam was estimated by the classical methods of strength of materials. The microstructural model was used for calculation of the phase deformation of the shape memory material. Simulation of stress-strain curves and phase deformation of a porous TiNi sample on cooling and heating under a constant stress has shown a good correspondence between the experimental data and the results of modeling.
\end{abstract}

\section{Introduction}

Owing to their low density, large surface area, high permeability and good biocompatibility, porous TiNi shape memory alloys (SMA) show great promise in bone implantations [1 - 3], actuators [4], damping devices [5] and even for hydrogen isotope separation and purification [6]. Development of techniques for control of porous structure [7] opens new prospects for applications. In order to make the most efficient use of this promising material, correct methods of calculation should be applied.

For calculation of the mechanical behavior of porous media methods of mechanics of composites are usually used [8]. D. Lagoudas [9] divides these methods into two groups: approaches involving application of micromechanical averaging techniques (like Mori-Tanaka method [10] or self-consistent method [11]) and the approaches based on the identification of a unit cell representative for the whole composite [12]. The second usually demands the finite element method to solve the corresponding boundary-value problem and deduce the effective properties.

Modeling of functional behavior of porous SMAs is complicated by phase deformation due to martensitic transformation. Among existing models one can single out the macroscopic [13-16] and the microstructural microstructural [17-19] ones. Macroscopic models are distinguished by few constants and high computation speed. Therefore they are usually used for simulation of porous SMAs in the frames of the mechanics of composites and the finite element method [9, 20-24]. At the same time macroscopic models have limited possibilities as they take the material structure into account but indirectly. For example, all the works mentioned above can describe only isothermal deformation of porous SMA. Microstructural models consider material structure with an account of the physical mechanisms of deformation of individual structural elements. This results in a better description of SMA behavior at different thermal and loading conditions. The flip side of microstructural models is that they take a lot of computing time and thus are poorly applicable for solving boundary-value problems.

In the present work we used an earlier developed microstructural model for calculation of the phase deformation of an SMA [25-27]. In these works it was shown that this model allowed describing such functional properties of SMA as pseudoelasticity, pseudoplasticity (ferroelastisity), transformation plasticity and shape memory effect. To avoid solving a complicated boundary-value problem for a representative element we supposed that its functional behavior as a whole is determined by that of the mostly strained region. Since the microstructural model is suitable for description of not only TiNi-based SMAs [28, 29], this approach seems to be proper for different porous SMAs with similar structure.

\section{Model}

In order to select a representative element for a porous SMA an analysis of longitudinal and transverse sections of samples fabricated by self-propagating high temperature synthesis [1] has been performed. It was supposed possible to approximate the structural elements of the porous sample by curved beams (Figure 1) characterized by the radius $R$, the central angle $\varphi$ and the

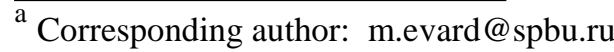


cross sectional dimensions $\tilde{b}$ and $\tilde{h}$ (the ligament width and height, respectively). It was assumed that the porous structure can be considered as a group of such curved beams. So the problem of the porous sample deformation was reduced to the task about the deformation of a cascade of curved beams. We supposed that the beams in the cascade were connected rigidly and the longitudinal displacement of the cascade on compression is realized along their common chord (Figure 2). Only the bending deformation of the beams was taken into account, buckling was not considered. It was also accepted that the bent line of a beam was a circular arc during all deformation process. An assumption of a rigid connection was realized by the demand that angular deflections of beam ending cross-sections be zero. So a statically indeterminate curved beam loaded along its chord by the force $P$ was examined for calculation of the bending moment $M$ and normal stress $\sigma$ and their relations with $R$ and $\varphi$ for an arbitrary cross-section corresponding to the angle $\varphi=\varphi^{*}$. It was found that the maximum normal stress $\sigma_{\max }$ was in the middle cross-section at $\varphi^{*}=\pi / 2$. For a beam with rectangular cross-section with width $b$ and height $h$

$$
\sigma_{\max }=-\frac{P}{b h}+\frac{2 P\left(\frac{\varphi}{2}-\sin \frac{\varphi}{2}\right)\left(R-\frac{h}{\ln \frac{R}{R-h}}\right)}{\varphi b h\left(R-\frac{h}{2}-\frac{h}{\ln \frac{R}{R-h}}\right)}
$$

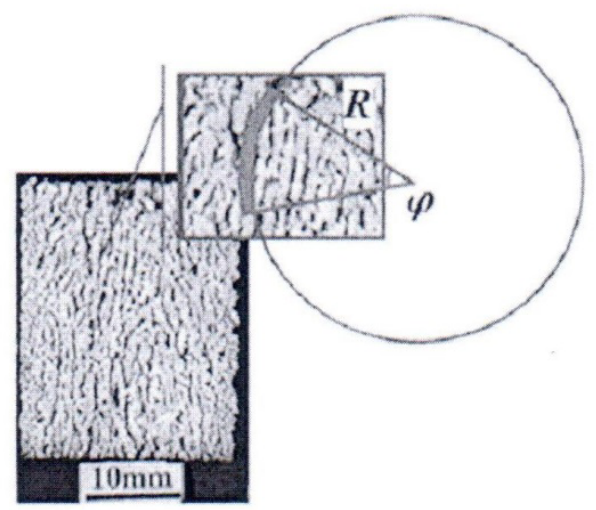

Figure 1. The longitudinal section of porous TiNi sample fabricated by self-propagating high temperature synthesis [1] and selected representative element with the radius $R$ and the central angle $\varphi$.

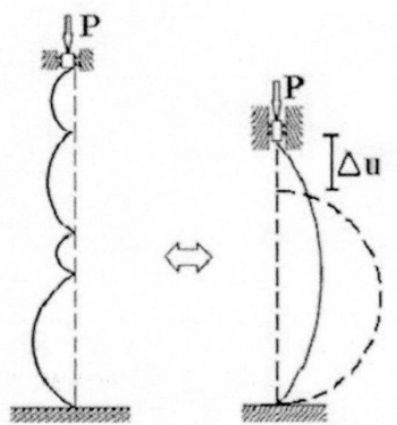

Figure 2. The scheme of a representative volume.
Modeling was carried out step by step. Given the force increment $\Delta P$ we obtained the increment of the maximum stress $\Delta \sigma_{\max }^{(i)}$ for $i$-th beam in the cascade. Then using the microstructural model $[25,26]$ we found the increment of deformation $\Delta \varepsilon^{(i)}$. Substituting this value into the formula for the deformation of a small curved beam we calculated the new values $R^{(i)}, \varphi^{(i)}$ of the beam radius and the arc angle.

The longitudinal displacement of the $i$-th beam along its chord is

$$
\Delta u^{(i)}=2 R^{(i)} \sin \frac{\varphi^{(i)}}{2}-2 R^{(i) \prime} \sin \frac{\varphi^{(i)^{\prime}}}{2} .
$$

Here $R^{(i)}$ and $\varphi^{(i)}$ are the beams radii and arc angles on the previous step (the initial values at the first step).

Increment of the deformation of the whole sample is

$$
\Delta \mathrm{E}=\frac{\sum_{i} n^{(i)} \Delta u^{(i)}}{\sum_{i} n^{(i)} l_{0}^{(i)}}
$$

where $n^{(i)}$ is a number of beams with $R^{(i)}$, and $\varphi^{(i)}$, in the cascade, $l_{0}^{(i)}$ is the initial chord length of the $i$-th beam.

Determine the macroscopic stress as

$$
\Sigma=\frac{\tilde{P}}{S(1-p)^{2}},
$$

where $\tilde{P}$ is the force applied to the whole sample, $S$ is the cross-section area of the sample, $p$ is the porosity measured by the random secant method. Then the relation between the local force $P$ applied to the cascade and the macroscopic stress $\Sigma$ can be written as

$$
\Sigma=\frac{P}{\tilde{b} \widetilde{h}(1-p)} \text {. }
$$

We supposed that if the local stress $\sigma_{\max }^{(i)}$ in the $i$-th beam reaches some critical value $\sigma^{*}$ this beam fails. This leads to an increase of the total deformation of the sample by the value

$$
\Delta \mathrm{E}=\frac{l^{\prime(i)}}{\sum_{i} n^{(i)} l_{0}^{(i)}},
$$

where $l^{\prime(i)}$ is the $i$-th chord length on the step, at which failure occurred. This failure also results in an increase of the stress applied to the rest of the sample. As the real sample can be considered as a set containing large number $k$ of parallel connected cascades we considered that when a beam failure occurs the current macroscopic stress value $\Sigma^{*}$ on the next step should be multiplied by $k /(k-1)$ :

$$
\Sigma=\Sigma^{*} \frac{k}{k-1} \text {. }
$$

In this way we indirectly took into account the complicated structure of the porous sample.

\section{Simulation}

\subsection{Determination of model parameters}

More than 100 measurements based on Figure 1 [1] were carried out to determine characteristic dimensions of beams - radii $R$ and central arc angles $\varphi$. The beams for the representative cascade were chosen by the way to reproduce the real structure of the sample. The parameters selected for modeling are presented in the Table 1. No significant differences of the beam cross- 
section dimensions were found, so we used $\tilde{b}=1.4 \mathrm{~mm}$, and $\tilde{h}=0.7 \mathrm{~mm}$.

Table 1. Parameters of the beams cascade.

\begin{tabular}{|c|c|c|c|c|c|}
\hline$R, \mathrm{~mm}$ & 1.7 & 2.9 & 4.6 & 7.1 & 11.9 \\
\hline$\varphi, \mathrm{rad}$ & 1.5 & 1.2 & 1 & 0.9 & 0.6 \\
\hline $\begin{array}{c}\text { number of } \\
\text { beams in } \\
\text { the cascade }\end{array}$ & 28 & 10 & 3 & 28 & 16 \\
\hline
\end{tabular}

The following values of the microstructural model were used: the characteristic temperatures of the transformation $M_{f}=347 \mathrm{~K}, M_{s}=330 \mathrm{~K}, A_{s}=362 \mathrm{~K}$, $A_{f}=379 \mathrm{~K}$, the latent heat of the transformation $q_{0}=-160 \mathrm{MJ} / \mathrm{m}^{3}$. Microplastic deformation (accommodation plasticity) and plastic deformation were also taken into account.

Calculations were made for the porosity $p=61 \%$, the critical stress $\sigma^{*}=700 \mathrm{MPa}$, number of parallel elements $k=102$.

\subsection{Results of modeling}

The stress-strain diagrams for the sample in austenitic and martensitic states as well as the experimental curves ${ }^{a}$ are presented on Figure 3. The polygonal character of the model curves is due to the consecutive failure of the "weak" beams.

Figure 4 illustrates the transformation plasticity and the shape memory effect at cooling and heating under a constant compressive stress. It was revealed that the significant irrecoverable deformation at cooling and heating under the stress $200 \mathrm{MPa}$ is induced not only by the accommodation and active plasticity but also by the failure of "weak" beams.

One can see that results of modeling both for isothermal and non-isothermal conditions are in a good correspondence with the experimental data of S. Belyaev and N. Resnina ${ }^{a}$.

\section{Conclusions}

The proposed model based on the elementary theory of bent beams and the microstructural model of SMA deformation allows describing compression stress-strain diagrams of the porous TiNi sample in the austenitic and in the martensitic state. This model also describes the strain accumulation on cooling of a specimen under a constant compression stress (transformation plasticity effect) and its reverse on heating (shape memory effect).

Generally, the approach under consideration is suitable for different types of primary elements of porous SMA and different kinds of loading. Combination of a proper mechanical problem and the microstructural model should give a possibility to calculate functional properties for various smart porous structures with different types of pores and ligaments [22, 30].

a The experimental results kindly presented by S. Belyaev and N. Resnina are to be published soon. a)

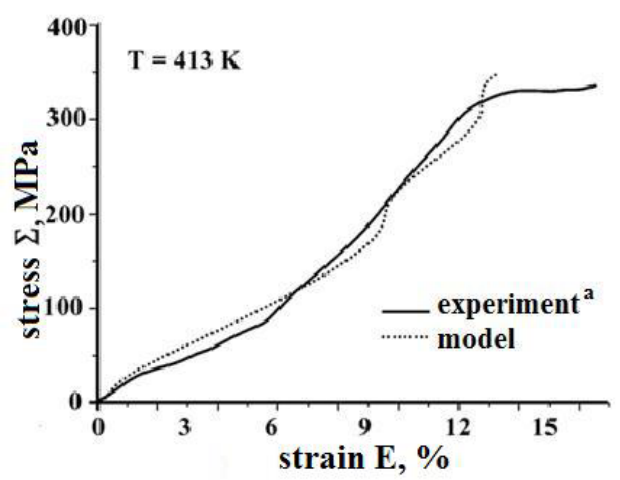

b)

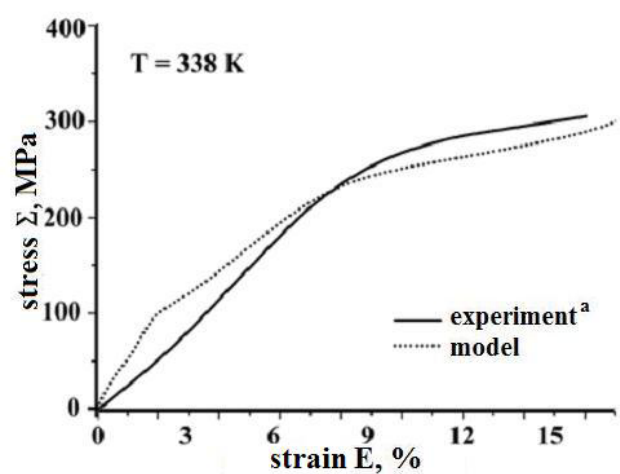

Figure 3. Stress strain diagrams for compression at $413 \mathrm{~K}(a)$ and $338 \mathrm{~K}(b)$ : calculation - solid lines; experiments ${ }^{\mathrm{a}}$ - dot lines.

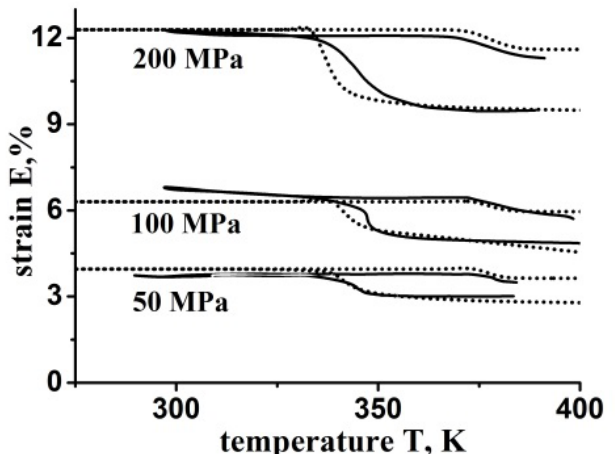

Figure 4. Dependences of the macroscopic strain $\mathrm{E}$ on temperature at cooling and heating under a constant stress 50, 100 and $200 \mathrm{MPa}$ : calculation - solid lines; experiments ${ }^{\mathrm{a}}$ — dot lines.

\section{Acknowledgements}

This research was supported by the grants of Russian Foundation of Basic Research 15-01-07657 and 15-0805-021.

The authors acknowledge Prof. S. Belyaev and Dr. N. Resnina for presented experimental results to be published soon.

\section{References}

1. B.Y. Li, L.J. Rong, Y.Y. Li, V.E. Gjunter, Acta Materialia, 48, 3895-3904 (2000) 
2. V.I. Itin, V.E. Gjunter, S.A. Shabolovskaya, R.L.C. Sachdeva, Materials Characterization, 32, 179-187 (1994)

3. A. Bansiddhi, T.D. Sargeant, S.I. Stupp, D.C. Dunand, Acta Biomaterialia, 4, 773-782 (2008)

4. A.P. Jardine, Patent US20140157768 A1 (2014)

5. Y. Zhao, M. Taya, H. Izui, Int. J. Solids Struct., 43, 2497-2512 (2006)

6. J.B. Zhou, L.P. Gao, K.S. Wang, J. Rare Earth, 23, 449-451 (2005)

7. Y.P. Zhang, D.S. Li, X.P. Zhang, Scripta Materialia, 57, 1020-1023 (2007)

8. Mechanics of Composite Materials: Recent Advances (Ed. by Z. Hashin, C.T. Heracovich), 1983

9. P.B. Entchev, D.C. Lagoudas, Mechanics of Materials, 34, 1-24 (2002)

10. T. Mori, K. Tanaka, Acta Metall., 21, 571-574 (1973)

11. A.V. Hershey, J. Appl. Mech., 21, 236-240 (1954)

12. S. Nemat-Nasser, M. Hori, North-Holland Series in Applied Mathematics and Mechanics, 37 (1993)

13. K. Tanaka, S. Kobayashi, Y. Sato, Int. J. Plasticity, 2, 59-72 (1986)

14. D.C. Lagoudas, Z. Bo, M.A. Qidwai, Mech. Compos. Mater. Struct., 3, 153-179 (1996)

15. I.V. Mishustin, A.A. Movchan, Mechanics of Solids, 49, 27-39 (2014)

16. F. Auricchio, L. Petrini, Int. J. Numer. Anal. Met., 61, 807-836 (2004)

17. E. Patoor, A. Eberhardt, M. Berveiller, J. de Phys. IV. C1, 6, $277-292$ (1996)

18. Q.-P. Sun, C. Lexcellent, J. de Phys. IV. C1, 6, 367 -375 (1996)

19. M. Huang, L.C. Brinson, JMPS, 46, 1379-1409 (1998)

20. P.B. Entchev, D.C. Lagoudas, Mechanics of Materials, 36, 865-913 (2004)

21. M.A. Qidwai, P.B. Entchev, D.C. Lagoudas, V.G. DeGiorgi, Int. J. Solids Struct., 38, 8653-8671 (2001)

22. Y. Zhao, M. Taya, Y.S. Kang, A. Kawasaki, Acta Materialia, 53, 337-343 (2005)

23. S. Nemat-Nasser, Y. Su, W.-G. Guo, J. Isaaks, JMPS, 53, 2320-2346 (2005)

24. M. Panico, L.C. Brinson, Int. J. Solids Struct., 45, 5613-5626 (2008)

25. M.E. Evard, A.E. Volkov, JEMT, 121, 102 (1999)

26. A.E. Volkov, M.E. Evard, K.V. Red'kina, A.V. Vikulenlov, V.P. Makarov et al., JMEP, 23, 2719 (2014)

27. A.E. Volkov, M.E. Evard, F.S. Belyaev, in Shape memory alloys: properties, technologies, opportunities (Ed. by N. Resnina, V. Rubanik), 2015

28. M.E. Evard, A.E. Volkov, Proc. of SPIE, 3345, 178183 (1997)

29. M.E. Evard, A.E. Volkov, Proc. of SPIE, 3687, 330334 (1999)

30. S. Arnaboldi, P. Bassani, F. Passaretti, A. Redaelli, A. Tuissi, JMEPEG, 20, 544-550 (2011) 